\title{
DRSIR: A Deep Reinforcement Learning Approach for Routing in Software-Defined Networking
}

\author{
Daniela M. Casas-Velasco, Oscar Mauricio Caicedo Rendon, and Nelson L. S. da Fonseca
}

\begin{abstract}
Traditional routing protocols employ limited information to make routing decisions which leads to slow adaptation to traffic variability and restricted support to the quality of service requirements of the applications. To address these shortcomings, in previous work, we proposed RSIR, a routing solution based on Reinforcement Learning (RL) in SoftwareDefined Networking (SDN). However, RL-based solutions usually suffer an increase in the learning process when dealing with large action and state spaces. This paper introduces a different routing approach called Deep Reinforcement Learning and SoftwareDefined Networking Intelligent Routing (DRSIR). DRSIR defines a routing algorithm based on Deep RL (DRL) in SDN that overcomes the limitations of RL-based solutions. DRSIR considers path-state metrics to produce proactive, efficient, and intelligent routing that adapts to dynamic traffic changes. DRSIR was evaluated by emulation using real and synthetic traffic matrices. The results show that this solution outperforms the routing algorithms based on the Dijkstra's algorithm and RSIR, in relation to stretching (stretch), packet loss, and delay. Moreover, the results obtained demonstrate that DRSIR provides a practical and viable solution for routing in SDN.
\end{abstract}

Index Terms-Deep Reinforcement Learning, Routing, Software-Defined Networking

\section{INTRODUCTION}

$\mathbf{R}$ OUTING is the network function that determines the end-to-end path between a source and a destination node. Traditional routing protocols usually make decisions based on shortest path calculation with limited additional information, this leads to slow adaptation to traffic variability and restricts its support for meeting Quality of Service (QoS) requirements. Moreover, the continuous growth of the Internet and the diversity of applications running on it have led to significant challenges affecting the efficiency of decisions based on limited information. On the other hand, the combination of Software-Defined Networking (SDN) and Machine Learning (ML) can help overcome such shortcomings. SDN provides opportunities to improve networks in relation to programmability, global view, logically centralized control, and decoupling of network control and packet forwarding. ML techniques provide intelligence to SDN, which enables it to learn autonomously to make optimal routing decisions adaptable to traffic variations.

Some papers [1]-[6] have shown to improve network routing by leveraging SDN capabilities, but they do not exploit network information intelligently. Other proposals [7]-[12] have used supervised ML techniques to optimize existing

D. M. Casas-Velasco, and N. L. S. da Fonseca are with the Institute of Computing, University of Campinas, Brazil. e-mail: danielac@1rc.ic.unicamp.br and nfonseca@ic.unicamp.br.

O. M. Caicedo is with the Department of Telematics, Universidad del Cauca, Popayán, Colombia. e-mail: omcaicedo@unicauca.edu.co. routing strategies, but the training of these algorithms is based on labeled datasets obtained from the operation of traditional routing protocols, which demands high computational complexity and makes the routing decisions dependent on limited information

The solutions in [13]-[17] have employed Reinforcement Learning (RL) to optimize the selection of routing algorithms. Compared with supervised ML techniques, RL learns by trial and error in the interaction with the environment, and, thus, does not depend on labeled datasets. Moreover, optimization targets (e.g., throughput and delay) can be easily adjusted by the definition of reward functions. In previous work [18], we proposed an approach called the Reinforcement Learning and Software-Defined Networking for Intelligent Routing (RSIR), which employs link-state metrics for routing in SDN. Results showed that RSIR outperforms the Dijkstra-based routing. Nonetheless, RL-based solutions usually can have a significant increase in the learning process when handling large action and state spaces, requiring the RL-agent to make several interactions with the environment to converge towards reliable estimations [19]. Various papers [20]-[30] have explored the use of Deep Reinforcement Learning (DRL) techniques to cope with the limitations of RL-based routing solutions. However, these papers focus on optimizing delay and neglect other performance metrics such as loss and throughput. Moreover, the use of link metrics to build a path can be restrictive, since decisions based on local (link) information disregard the state of other links on the path to the destination node.

In this paper, we take a step further towards the goal of an efficient and intelligent routing scheme in SDN by introducing a novel approach, called the Deep Reinforcement Learning and Software-Defined Networking for Intelligent Routing (DRSIR), which combines Deep Learning (DL) and RL to overcome the shortcomings of RL-based routing solutions. DRSIR introduces a model-free DRL-based algorithm that uses path-state metrics and the global view and control offered by SDN to compute and install optimal routes proactively in forwarding devices, thus allowing adaption to dynamic traffic changes without prior knowledge of the underlying network. Using path-state metrics enables the reduction of knowledge abstraction needed by the routing agent since this approach directly explores different path options instead of link state information. The DRSIR algorithm calculates optimal routes using the Target and Online Neural Networks (NNs) that allows the DRL-agent to reduce the error in estimations based on path information. Moreover, the DRL-agent uses Experience Replay Memory to accelerate the learning process. Results show that DRSIR outperforms RSIR and other four variations of the Dijkstra's algorithm in relation to stretch, 
link throughput, delay, and packet loss. The results show that DRSIR is a promising solution for cognitive routing in SDN.

The contributions of this paper are:

- A novel routing algorithm based on Deep Q-Learning, Experience Memory Replay, Target Neural Network, and Online Neural Network that considers path-state metrics to define optimal routes in SDN;

- A prototype of the proposed algorithm.

The remainder of this paper is organized as follows. Section II presents a brief background for the relevant concepts. Sections III and IV detail our approach by introducing the DRL-based routing architecture, the DRSIR agent, and the routing algorithm. Section V presents the DRSIR prototype and its evaluation results. Section VI compares the DRSIR to existing approaches. Section VII presents conclusions and suggestions for future work.

\section{BACKGROUND}

In RL models, a Markov Decision Process (MDP) formulation defines a set of states and actions $(S, A)$, and a reward model, hereafter, called environment. In RL, a learning entity (an agent) interacts with the environment by iterating over states and selecting actions to be made. In the interaction, the agent receives a reward value for each action applied [31]; this expresses its impact on the system performance. It aims at learning a policy that optimizes the cumulative reward. Thus, the agent learns by trial and error, observing the reward obtained by the different actions performed. The learning of an optimal policy $\pi$ involves the estimation of a value function (e.g., action-value function), which indicates the goodness of an action $A_{t}$ performed when the MDP is in a state $S_{t}$ under policy $\pi$ [32].

RL algorithms can be either model-based, or model-free [33]. For model-based algorithms, the agent employs a model of the environment (i.e., a function that predicts state transitions and rewards); this model defines how it should operate in finding a solution. Such algorithms require a ground-truth model of the environment that is usually not available. On the other hand, model-free algorithms do not need such a model of the environment to find an optimal policy; they learn to estimate the expected reward for different actions either by learning a value function approximation from which the policy is inferred or by directly learning the policy function that maps states to actions. RL algorithms can also be classified as either off-policy or on-policy [31]. Off-policy refers to the fact that the algorithm does not follow the same policy for sampling (i.e., selecting an action for the current state) and updating its value function. Instead, it chooses the action corresponding to the best reward of actions available for updating the value function. In on-policy algorithms, the policy used for updating and acting is the same.

A well-known RL algorithm is Q-learning [34], which is model-free and off-policy. The action-values are represented by a state transition table (Q-table), where each state-action pair $\left(S_{t}, A_{t}\right)$ has an entry called Q-value $Q\left(S_{t}, A_{t}\right)$. To update the Q-value, Q-learning uses Equation 1.
$Q_{t+1}\left(S_{t}, A_{t}\right)=Q_{t}\left(S_{t}, A_{t}\right)+\alpha \cdot\left[R_{t}+\min _{\mathrm{A}} Q_{t}\left(S_{t+1}, A\right)-Q_{t}\left(S_{t}, A_{t}\right)\right]$

where:

- $R_{t}$ is the reward retrieved by the RL-agent in time $t$ when executing an action $A_{t}$ at a given state $S_{t}$.

- $\alpha \in[0,1]$ is the learning rate that determines the weight of the newly gained information in relation to what was previously available.

- $\gamma \in[0,1]$ is the discount factor that determines the importance of future rewards.

The expression in square brackets is the difference between the target Q-value computed by $R_{t}+\min _{\mathrm{A}} Q_{t}\left(S_{t+1}, A\right)$ and the current Q-value $Q_{t}\left(S_{t}, A_{t}\right)$ for a pair of state-action $\left(S_{t}, A_{t}\right)$. When $\alpha=0$, the RL-agent does not to learn from the latest $\left(S_{t}, A_{t}\right)$ pair, when $\alpha=1$ the RL-agent retains the learned information by considering the immediate reward $R_{t}$ from the pair $\left(S_{t}, A_{t}\right)$. Moreover, a value of $\gamma=0$ enables the RL-agent to consider only the current reward $R_{t}$, while a factor $\gamma=1$ makes possible for the RL-agent to consider future rewards.

The learning process of $\mathrm{RL}$ can be quite low when the state and action spaces are large, and Deep Learning (DL) has jointly been employed with RL to accelerate the learning process. Such combination is denominated Deep Reinforcement Learning (DRL), and it uses function approximators such as NNs to extract knowledge from visited states. The use of DL makes it possible for RL to obtain solutions that were intractable when the latter was employed alone [35]. Deep Q-learning (a.k.a. Deep Q-Network (DQN) algorithm) is a DRL technique conceived as an extension of Q-learning. During the training of the DQN agent, the $\mathrm{NN}$ generalizes the approximation of the Q-value function rather than using a value from the $\mathrm{Q}$-table. The $\mathrm{NN}$ receives a state as input and maps it onto all possible Q-value actions.

\section{DRL-BASED ROUTING ARCHITECTURE}

In this section, we introduce a DRL-based routing architecture. As illustrated in Figure 1, the architecture is composed of the Topology Module, the Monitoring Module, the Processing Module, the Routing Module, and the Installation Module. These modules i) gather raw network data (e.g., port statistics) from the forwarding devices located in the Topology Module (1 1 in the figure), ii) process the raw data to compute path-state metrics (2), iii) explore and learn information on the state of a path (path-state) to compute optimal paths for all sourcedestination nodes in the network $(3)$, iv) retrieve routing path information (4); and v) execute installation of flows in the forwarding devices according to the paths computed (5).

The Topology Module represents the set of forwarding devices (i.e., switches) in the underlying network. This module responds to query requests with statistical information gathered at the ports of the switches during running time. The Monitor Module gathers this statistical information and produces a topology map by employing the Network Discovery and Network Statistics functions. The Network Discovery function exchanges messages with the Topology Module containing feature information. With the information received, the 


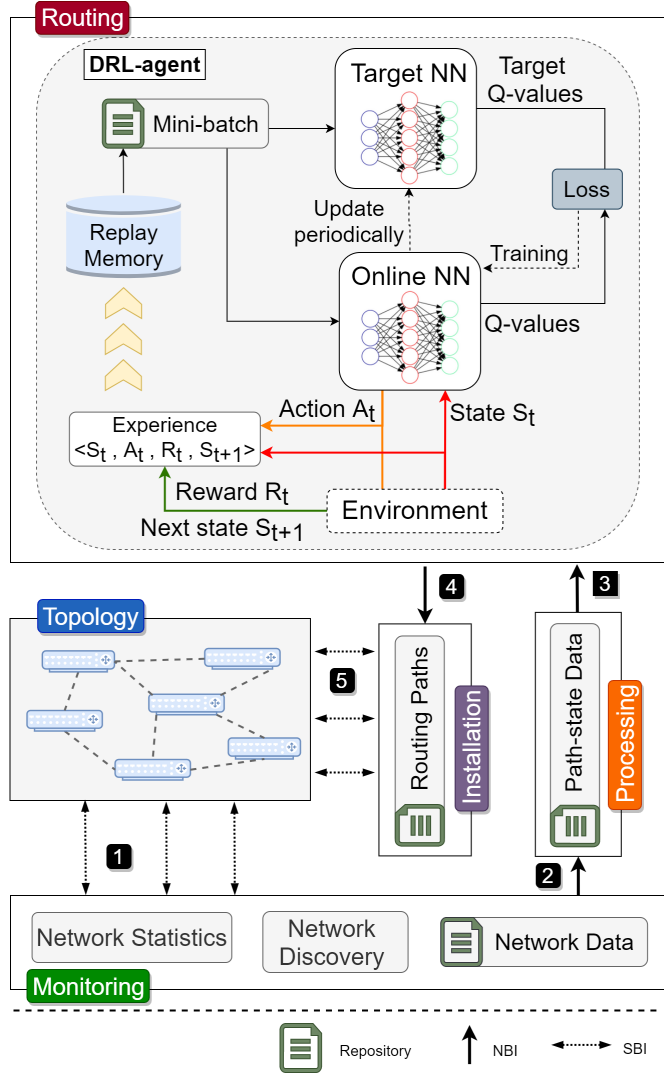

Fig. 1: DRL-based Routing Architecture

Network Discovery function maps switch ports to the ports of its neighboring switches and furnishes the topology as a set of tuples (i.e., switch id, port id, neighbor switch id, neighbor port id) in the Network Data Repository. The Monitor Module detects topology changes related to nodes and links by periodically gathering topology information at each Change Detection Time $\left(t_{c h a d}\right)$. The Network Statistics function is responsible for gathering statistical information by exchanging state messages with each switch at every $t$ seconds. The replied messages from the Topology Module are stored in the Network Data Repository and provide an updated global view of the network.

The Processing Module retrieves raw data from the Network Data Repository, and then computes metrics that characterize the state of a path (path-state metrics), which is stored in the Path-state Data Repository as a set of entries containing information about the source, destination, and a tuple of metrics. The path-state metrics serve as input features for the Routing Module which employs DRL to learn the network behavior and create an optimal routing plan. The Routing Module hosts a DRL-agent using DQN (as described in subsection IV-A). The DRL agent learns and calculates paths by running the DRLbased routing algorithm (as described in subsection IV-B) during a Learning Time $\left(t_{\text {lear }}\right)$. The Routing Module passes the routing plan to the Installation Module and saves it in the Routing Paths Repository. Each entry in such repository is a tuple representing the source, destination, and selected path.

The Installation Module retrieves path information from the
Routing Paths Repository and installs/updates flow rules in the switches. The Installation module proactively populates the flow tables of the switches (i.e., prior to all traffic matches), which takes place in a time interval that depends on the number of forwarding tables and the number of flow entries to update, called Installation Time $\left(t_{\text {inst }}\right)$.

\section{DRSIR}

DRSIR is a DRL-based routing approach for SDN. It employs the architecture presented in Figure 1, which uses metrics describing the states of the paths to explore, learn, and exploit potential paths for all the source-destination pairs. The metrics used are path bandwidth, path delay, and path packet loss ratio. In this section, we detail the DRL-based agent and the DSIR routing algorithm.

\section{A. DRSIR Agent}

Figure 1 depicts the DQN agent hosted in the Routing Module, which also hosts the environment for training the agent on the basis of the Action and State Spaces and the pathstate information provided by the Processing Module. The reward value $R_{t}$ is computed for action $A_{t}$ at state $S_{t}$, allowing the agent to find the next state $S_{t}$ to be visited. The Reward function gives the path cost quantified by path-state metrics. The DQN agent learns the policy that selects actions which will minimize the reward function. The DRSIR agent learns to avoid high delay and loss ratios and prioritize paths with large available bandwidth for making intelligent routing decisions. The DQN-agent includes an Online NN, a Target NN, and a Replay Memory database [36] to minimize the reward, learn fast, and enhance stability in learning.

Next, we give details about the state and action spaces, the reward function, the optimal policy, the Replay Memory, the Online and Target NNs, and the exploration method used by the DRSIR DQN agent.

1) State Space $(S)$ : The State Space is the set of states that the DQN agent can observe. Each state corresponds to a source-destination pair of nodes that communicates over the network. Given a network topology with $N$ nodes, the number of source-destination pairs is given by the k-permutations $P(N, j)$ of $N$, where $j=2$. Therefore, the size of the State Space is $|S|=|P(N, j)|=N ! /(N-2)$ !. The Network Discovery function builds a map of the topology of the network stored in the Topology Module, and the DQN agent builds the State Space from the topology map.

2) Action Space (A): The Action Space is the set of actions that can be taken on the states in the State Space. Each action $A_{t} \in[0, \ldots, k]$ corresponds to the selection of a specific endto-end path $p_{i} \in\left[p_{0}, \ldots, p_{k}\right]$ for a given state $S_{t}$. Therefore, the DQN agent can select one path among a list of $k$ candidate paths that connect a source and a destination node. DRSIR defines the $k$ shortest paths for each source-destination pair, computed by the Processing Module.

3) Reward Function: A Reward value is computed on the basis of path-state metrics and gives the cost of a potential path in the Action Space. DRSIR uses the Monitor and Processing Modules located at the SDN controller to calculate the cost of 
all potential paths in the Action Space. First, the Processing Module computes the instantaneous throughput $b w a_{l i n k}$, delay $d_{\text {link }}$ and packet loss ratio $l_{\text {link }}$ of all links considering the number of packets that passed through the switch port connected to the link. At each port, the SDN controller periodically samples the number of bytes transmitted and received. By comparing the retrieved values at two instants, it is possible to compute the instantaneous throughput. After sending a state-message to the Topology Module at time $t_{1}$, a reply message is recovered containing the received bytes, $b_{t_{1}}$. Then, after a period $p$, another request-stats message is sent, and the number of bytes received $b_{t_{2}}$ is retrieved from its reply message. The expression $b w u_{l i n k}=\left[\left(b_{t_{2}}-b_{t_{1}}\right) / p\right]$ gives the instantaneous throughput, where $p$ is the duration of the sampling interval. The available link bandwidth $b w a_{\text {link }}$ is thus computed as the difference between the link capacity cap $_{\text {link }}$ and the instantaneous throughput of the link bwu $u_{\text {link }}$, $b w a_{\text {link }}=c_{a p} p_{\text {link }}-b w u_{\text {link }}$. The expression $l_{\text {link }}=\left(b t x_{t_{1}}-\right.$ $\left.b r x_{t_{2}}\right) / b t x_{t_{1}}$ gives the instantaneous loss ratio, where $p$ is the period between the arrival of reply messages containing the transmitted bytes $b t x_{t_{1}}$ and $b r x_{t_{2}}$ at times $t_{1}$ and $t_{2}$, respectively.

The computation of the instantaneous delay value follows the method described in [37], which uses the Link Layer Discovery Protocol (LLDP) [38] and OpenFlow messages [39]. The Processing Module sends an LLDP message via the SDN controller $c_{0}$, which goes through the path $c_{0}-s_{i}-s_{j}-c_{0}$, with $s_{i}$ and $s_{j}$ being the switches connected by the link $\left(s_{i}, s_{j}\right)$. The time elapsed between transmission and reception of the LLDP message is the difference between the timestamp values $\left(d_{l l d p_{c i j}}\right)$. The time taken by the message to go from $c_{0}$ to the $s_{i}$ port $\left(c_{0}-s_{i}\right)$ is estimated as half the time elapsed between the transmission and reception of the OpenFlow echo_request and echo_reply messages sent by $c_{0}$ to $s_{i}$. A similar procedure is used to estimate the time elapsed as the message goes from $s_{j}$ to $c_{0}$. The expression $d_{s i-s j}=d_{l l d p_{c i j}}-d_{c_{0}-s_{i}}-d_{c_{0}-s_{j}}$ gives the instantaneous delay in the link $\left(s_{i}, s_{j}\right)$. For a given path $P \in A$, the Processing Module uses the bwalink, $d_{\text {link }}$ and $l_{\text {link }}$ of each link $i \in P$, to compute the $b w a_{\text {path }}$, delay $d_{\text {path }}$ and packet loss ratio $l_{\text {path }}$ employing Equations 2, 3, and 4:

$$
\begin{gathered}
b w a_{\text {path }}=\min _{i \in \mathrm{P}}\left(b w a_{\text {link }_{i}}\right) \\
d_{\text {path }}=\sum_{i \in P} d_{\text {link }_{i}} \\
l_{\text {path }}=1-\left[\prod_{i \in P}\left(1-l_{\text {link }_{i}}\right)\right.
\end{gathered}
$$

The Reward Function is inversely proportional to the path available bandwidth $b w a_{p a t h}$ and directly proportional to the path delay $d_{\text {path }}$ and the path packet loss ratio $l_{\text {path }}$. To avoid that one of the path-state metrics have more influence than the others in the learning process, we normalize the metrics values using the Min-Max technique [40], which rescales the range of the values of the metrics to a range with values in an arbitrary interval $[a, b]$. The Reward Function is defined in Equation 5, where $b \hat{w} a_{p}, \hat{d}_{p}, \hat{l_{p}}$ are the normalized values of the path available bandwidth, delay, and loss ratio, respectively, and the values $\beta_{1}, \beta_{2}$ and, $\beta_{3} \in[0,1]$ are tunable parameters useful to provide a weight value to a metric in the reward calculation.

$$
\hat{R}=\beta_{1} \cdot \frac{1}{b \hat{w a_{p}}}+\beta_{2} \cdot \hat{d_{p}}+\beta_{3} \cdot \hat{l_{p}}
$$

4) Target and Online Neural Networks: The DQN-agent employs two NNs, the Online and Target Networks. The Online NN estimates the Q-values on the current state $S_{t}$ (i.e., $\left.Q\left(s_{t}, A_{t}\right)\right)$, while the Target NN outputs the Q-values on the next state $S_{t+1}$ (see Equation 6). The Online NN is trained at each learning step to decrease the loss function (Equation 7). At the start of the learning process, the weights of the Target $\mathrm{NN}$ and those of the Online NN are the same. The values of these weights are temporarily frozen to enhance learning stability. During the training phase, the weights of the Target $\mathrm{NN}$ are periodically updated to match the Online NN after a pre-determined number of learning steps.

$$
\begin{gathered}
Q^{+}\left(S_{t}, A_{t}\right)=R_{t}+\gamma \cdot \min _{\mathrm{A}} Q\left(S_{t+1}, A_{t}\right) \\
\text { Loss }=\left(Q^{+}\left(S_{t}, A_{t}\right)-Q\left(S_{t}, A_{t}\right)\right)^{2}
\end{gathered}
$$

In DRSIR, the Online and Target NNs have the same structure, including an input layer, one or several hidden layers, and an output layer. For each state in the State Space, the DQN agent encodes each source and destination pair as a state. The input layer has one neuron that receives the state as the input of the NN. The output layer has $k$ neurons, i.e., one neuron for each of the $k$ actions in the Action Space $A$. Each neuron in the output layer estimates a $Q_{i}$-value associated with action $a_{i} \in A$. The number of hidden layers is defined by testing in Section V-D.

5) Replay Memory: The DQN-agent stores past decisions (experiences) in a dataset with entries of the form $S_{t}, A_{t}, R_{t}, S_{t+1}$ in the Replay Memory, which allows sampling batches of experiences and training offline on previously observed data. We use this dataset to reduce the number of interactions needed by the DQN-agent to learn [36]; minibatches can be sampled for this purpose, thus reducing the variance in learning updates [41].

6) Exploration Method: DRSIR uses the Decay $\varepsilon$-greedy exploration method [42]. This method employs a tunable parameter, $\varepsilon \in[0,1]$, to determine if the agent should exploit with a probability $p r=\varepsilon$ or explore with a probability $p r=$ $1-\varepsilon$. The $\varepsilon$ value is usually set to start at a maximum value $\varepsilon_{\max }$ and linearly decrease at a decay rate $\operatorname{decr}$ throughout the learning process (steps) until reaching a minimum value $\varepsilon_{\min }$, according to the expression $\varepsilon=\varepsilon_{\max }-($ steps $\times$ decr $)$. Such a decrease allows the DQN agent to go from an exploratory policy at the start of the learning process to a more exploitative policy as the learning process progresses [34]. The DQN agent follows Equation 8 to select the next action in a specific state. For each learning step, a random value $x \in[0,1]$ is generated. If $x<\varepsilon$, the agent exploits. Otherwise, the agent explores.

$$
\mathcal{A}=\left\{\begin{array}{l}
\min _{\mathrm{A}} Q_{t}\left(S_{t}, A\right), \quad \text { if } x<\varepsilon \\
\text { randomaction, }
\end{array}\right.
$$




\section{B. DQN-based Routing Algorithm}

The DRSIR introduces a routing algorithm that implements a learning process to find the best paths for all the node pairs in the network. Algorithm 1 receives the following parameters as input: i) the number of learning episodes $n$, ii) the maximum value of the $\varepsilon$ parameter, iii) the decay rate $d e c r$, the number of steps to start the training employing the dataset stored in the Replay Memory (Replay start size rss), iv) the frequency of steps in which the Target NN is updated (Target update frequency tup), v) the list of the " $k$ " paths per state $k_{\text {paths }}$, and vi) information on the state of the paths. The output is the set of best-rewarding routing paths for all pairs of nodes in the network. The path is formed by state-action pairs with the lowest values in the Q-table.

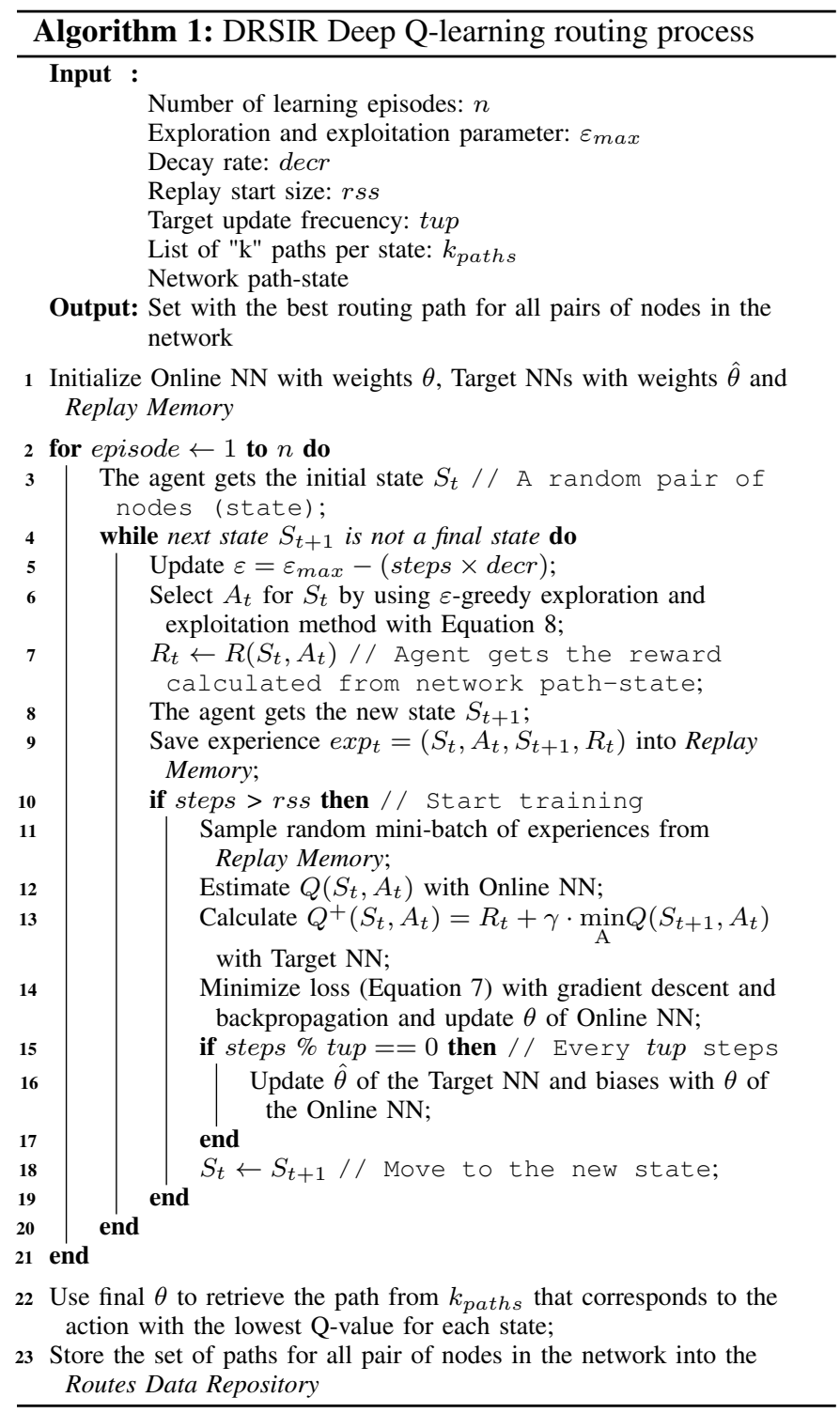

Algorithm 1 initializes the Online and Target NNs with weights $\theta$ and $\hat{\theta}$, respectively, as well as the Replay Memory (Line 1). The routing algorithm goes through episodes according to the steps in the loop from Line 2 to Line 21, starting in an initial random state $S_{t}$. For each episode, the DQN agent goes through a finite number of steps in an inner loop and reaches the next state $S_{t+1}$, the ending state (i.e., state after going through $m$-steps) (Lines 4 to 20). Therefore, a learning episode comprises a sequence of steps that correspond to the states between an initial state and a final state. Each step consists of selecting and performing an action, changing the state, and receiving a reward.

The inner loop (Line 4 to Line 20) is executed as follows. First, the DQN agent updates the value of the $\varepsilon$ parameter considering the decay rate decr (Line 5). It selects an action from the Action Space (selects $A_{t}$ for $S_{t}$ ), which is the selection of a path from the $k$ candidate paths for the current state by using the $\varepsilon$-greedy method (Line 6). Then, the DQN agent obtains a reward $R_{t}$ computed in the environment by using Equation 5 (Line 7). The DQN agent also gets the next state $S_{t+1}$ (the next pair source-destination) (Line 8). Finally, the experience $\exp _{t}=\left(S_{t}, A_{t}, S_{t+1}, R_{t}\right)$ is saved in the Replay Memory (Line 9).

The DQN agent stores the experiences in the Replay Memory, and verifies the number of steps to start the training of the Online NN, rss steps, (Line 10). When rss is reached, the DQN agent randomly takes a mini-batch from the $R e$ play Memory to train the Online NN (Line 11). Training using experiences allows the agent to learn after a fewer interactions than does an RL-agent. Then, the DQN agent estimates $Q\left(S_{t}, A_{t}\right)$ by using the Online NN (Line 12), and calculates the associated target values $Q^{+}\left(S_{t}, A_{t}\right)$ by using the Target NN (Line 13). After that, the gradient descent and backpropagation algorithms are used to adjust the weights and biases of the Online NN and to minimize the loss calculated by Equation 7 (Line 14). After the execution of tup steps and the storage of a certain number of experiences, the DQN agent updates the weights and biases $\hat{\theta}$ of Target NN with the weights and biases $\theta$ of the Online NN (Line 15 to Line 17). The DQN agent then moves to the next state (Line 18). Finally, after the DQN agent makes the transition, it uses the final $\theta$ value to retrieve the path from $k_{\text {paths }}$ that corresponds to the action with the lowest Q-value for each state (Line 22). Once the DQN agent finds the best path for all pairs of sourcedestination nodes, it stores them in the Routes Data Repository (Line 23). The Installation Module retrieves these best paths and installs them in the routing tables of the switches.

The worst-case complexity of Algorithm 1 is derived as follows. DQN relies on the use of RL and the generalization given by the NNs. In the worst case, the RL agent visits all the states in the State and Action spaces, which implies a complexity dependent on the size of the spaces. In DRSIR, the State Space size is $O\left(N^{2}\right)$ where $N$ is the number of source-destination pairs in the network. The size of the Action Space is limited to the $k$ potential paths associated with each state. The complexity for RL is, then, $O\left(k N^{2}\right)$. Since $k$ is a constant, the worst-case complexity is $O\left(N^{2}\right)$.

In DRSIR, the DQN agent learns from a reduced set of experiences by generalizing the past decisions. The great advantage of employing the Online and Target NNs in the DQN agent is the avoidance of visiting all the state-action pairs to converge to an optimal policy. The computational complexity for training the NNs is bounded by their architecture and the input samples size (i.e., states). As the architecture of the 
Online and Target NNs is fixed (i.e., the number of hidden layers, the input size, and the output size), then the maximum number of iterations for learning is $O(1)$. Therefore, the complexity of training the NN has an $O\left(N^{2}\right)$, where $N$ is the total number of states. We refer the reader to [43] for further explanation about the complexity of training NNs. Finally, the worst-case complexity of Algorithm 1 is $O\left(N^{2}\right)$.

\section{Evaluation}

This section presents the evaluation of DRSIR. Subsection $\mathrm{V}-\mathrm{A}$ depicts the test environment. Subsections V-B and V-C show the performance metrics and traffic generation procedure, respectively. Subsection V-D presents the set up of the learning parameters, and Subsection V-E discusses the results.

\section{A. Test Environment and Prototype}

The DRSIR was evaluated using several topologies; in this paper, the results are shown for 23-node and 48-node topologies. The 23-node topology mirrors the GÉANT topology [44], an European data network. GÉANT, in 2004, had 37 links, with $50 \%$ of them having $10 \mathrm{Gbps}$, $40 \%$ having 2.5 Gbps, and $1 \%$ having 155 Mbps. We scaled these capacities to $100 \mathrm{Mbps}, 25 \mathrm{Mbps}$, and $1.55 \mathrm{Mbps}$ due to limitations imposed by the resources available in the machines running the Mininet emulator. The 48-node topology was generated using the Barabasi-Alberth algorithm [45]; which has 60 links with equal capacities of $100 \mathrm{Mbps}$. In both topologies, each switch had a host that forwarded and received traffic. The Data Plane was developed using Mininet 2.2.2 [46] with Open vSwitches 2.3.1 and Python scripts running on an Ubuntu Server 14.04 Virtual Machine (VM) with 8 GB RAM.

We used the Application Program Interface (API) of the Ryu controller [47] to develop the Monitoring and Installation Modules with Openflow 1.3 [48] as the Southbound Interface (SBI) to communicate with the Topology Module. We developed the Processing Module and the DQN agent by using Python 3.5 with Tensorflow 2.2.0 [49], Numpy 1.16.4 [50], and the Pandas 0.22 library [51]. We used Comma-Separated Values (CSV) and JavaScript Object Notation (JSON) files to store information in the Repositories. Every module in the DRL-based architecture ran on an Ubuntu 16.04 VM with a Core i5-4690 processor and 10 GB RAM. The VMs used for this prototype were hosted on an Ubuntu Desktop 16.04 with an Intel Core i5-4690 and 16 GB RAM. These VMs communicated using the Transmission Control Protocol (TCP). The DRSIR prototype, as well as all test scripts, are available in [52].

\section{B. Performance Metrics}

Table I shows the most common performance metrics used to evaluate the routing proposals: the link throughput, link loss ratio, and link delay. Moreover, we evaluated the stretch of the paths that compares the length of a path to the theoretical shortest path [53]. We developed an application on the SDN controller, which computed the shortest path by implementing the Dijkstra algorithm with equal edge weights. We computed the performance metrics as explained in Subsection IV-A.
The DRSIR DQN-based routing is compared with that of the RSIR algorithm [18]. As RSIR uses link-state metrics, we have modified RSIR to use path-state metrics as does DRSIR to carry out a proper comparison. We compare DRSIR with different variations of the Dijkstra's algorithm using the instantaneous delay (Dijkstra delay $)$, instantaneous loss (Dijkstra $\left.{ }_{\text {loss }}\right)$, and link available bandwidth $\left(\right.$ Dijkstra $\left._{\text {bw }}\right)$ as edge weights. Moreover, DRSIR is compared to that of Dijkstrabased routing using the link metric defined by Equation 9, which considers all the mentioned link metrics in the computation of edge weight (Dijkstra $\mathrm{comp}_{\text {com }}$. In an attempt to conduct a fair comparison, all the routing variations of the Dijkstra's algorithm (developed and executed as applications on the SDN controller) and the RSIR algorithm were subject to the same traffic scenario applied to the DRSIR algorithm in the evaluated topologies.

$$
\text { weight }=\frac{1}{b w a_{l i n k}}+d_{l i n k}+l_{l i n k}
$$

\section{Traffic Generation}

In the emulation experiments, scripts for generating traffic using the iperf3 tool were developed. The scripts generated User Datagram Protocol (UDP) traffic for clients and servers on the hosts, allowing the setting of transmission rates specified by the employed traffic matrices. The elements of these matrices gave the traffic between pairs of nodes at different times of the day. Sixteen publicly available intra-domain traffic matrices [54] with values collected for the GÉANT topology were used for the 23-node topology. Fourteen traffic matrices were generated via the Modulated Gravity Model [55] for the 48-node topology; peak hours having high traffic intensity (from 7:00h to 14:00h).

\section{Learning Parameters Setup}

The widely used Adaptive moment estimation (Adam) optimizer [56] was employed by the DQN agent. The Adam optimizer improves the gradient descent algorithm allowing quick convergence in the training of deep networks. Moreover, the Adam optimizer is also straightforward to implement and requires little memory [57]. The Glorot uniform initializer [58] for weights initialization and the ReLu activation function [59] were employed by the DQN agent. These techniques have been shown to maintain variance values similar during the learning process in all NN layers.

For setting the learning parameters in the training of the DQN agent, the convergence of a minimized reward in episodic training was used as a parameter for comparison. Several preliminary tests were conducted to set the learning parameter values. Figure 2 shows the observed reward as a function of the following learning parameters: number of hidden layers $h l s$ in conjunction with convergence time, number of neurons in hidden layer neu, discount factor $\gamma$, number of steps to the start of training the Online NN (replay start size $r s s$ ), decay rate decr at which the DQN agent explores and exploits, the size of the mini-batch used to train the NNs, and the number of steps indicating the updating frequency of 


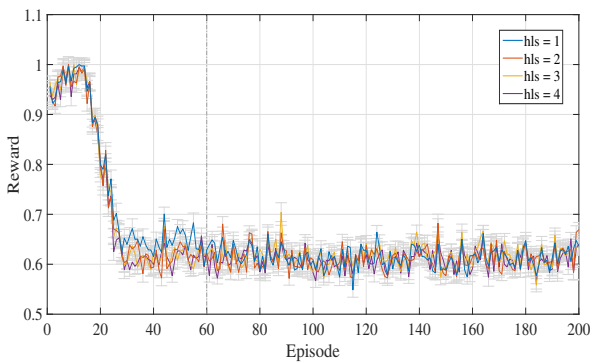

(a) Varying number of hidden layers $(h l s)$

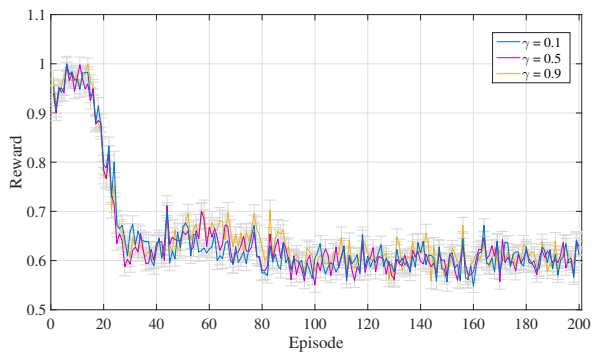

(d) Varying discount factor $(\gamma)$

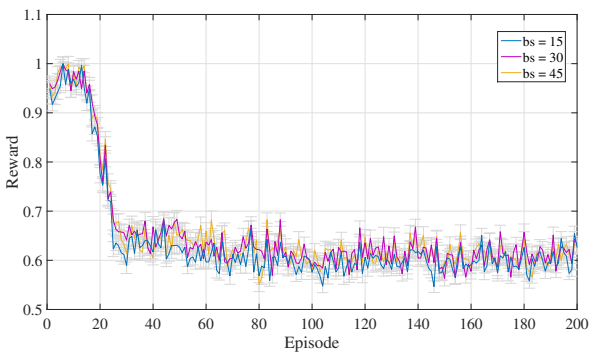

(g) Varying the mini-batch size $(b s)$

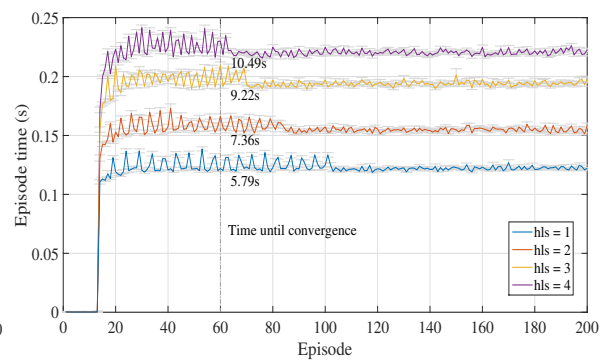

(b) Convergence time with different $h l s$

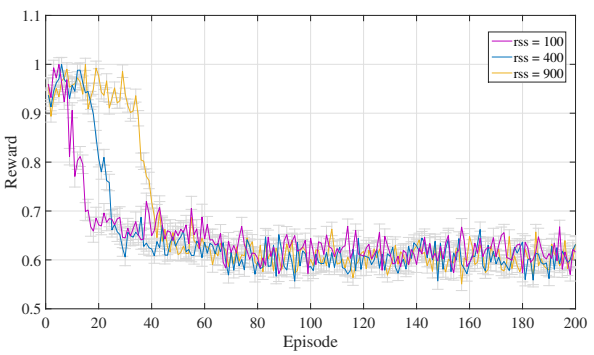

(e) Varying replay start size $(r s s)$

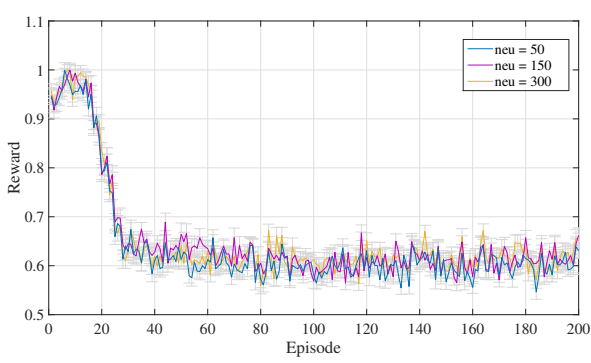

(c) Varying number of neurons (neu)

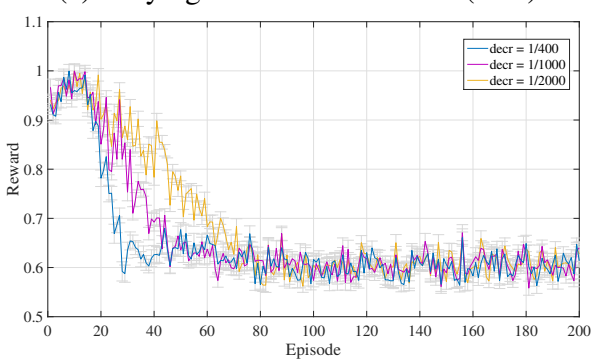

(f) Varying decay rate $($ decr $)$

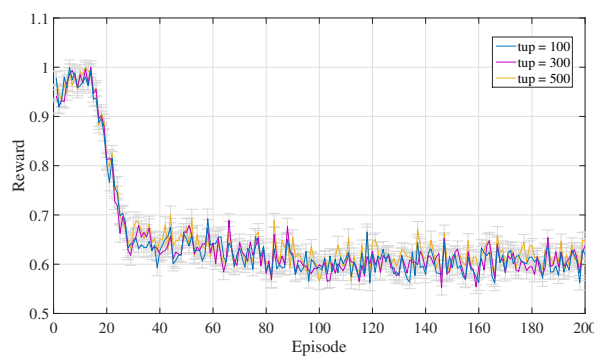

(h) Varying target update frequency (tup)

Fig. 2: Reward with respect to different learning parameters (number of neurons, $\gamma, r s s$, decr, $b s$, and tup)

the Target NN (target update frequency tup). For the sake of brevity, we only show three or four results for each parameter.

We run the Algorithm 1 to observe the returned reward in a single episodic training composed of 200 episodes with 30 steps per episode for each test, to identify the algorithm convergence to a minimized reward value as a function of the learning parameters. Figure $2 \mathrm{a}$ shows that after roughly the 60th episode, the reward no longer decreased significantly as a function of the number of hidden layers. Figure $2 b$ shows the impact on the convergence time of using one or more hidden layers until the 60th episode. With $h l s=1$, the produced reward is similar to that with other values of $h l s$ after convergence. However, it takes only $5.79 \mathrm{~s}$ to achieve these reward values, while with $h l s=2,3$ and 4 , the convergence time is $1.6 s, 3.46 s$ and $4,73 s$ higher than that. Thus, the Target and Online NNs were defined with a single hidden layer, which has been proven to be sufficient to approximate any function correctly [60]. Figure 2c shows that a hidden layer with 50 neurons obtains a slightly lower reward than hidden layers with 150 or 300 neurons. Moreover, a larger number of neurons requires more extended training periods.

Figure $2 \mathrm{~d}$ depicts the reward as a function of $\gamma$. For $\gamma=0.1$, the DQN agent slightly decreases the reward, but the value is still close to those given by $\gamma$ equals to 0.5 and 0.9 . In Figure 2e, the rss parameter indicates the number of steps before starting to exploit the knowledge obtained from experience when training the NNs, e.g., the value rss $=100$ means that the training of the NNs started after 100 steps. Results show that a value of $r s s=400$ quickly causes the agent to start minimizing the reward, and achieving a slightly lower reward than when rss is 100 or 900 . For the exploitation and exploration method, $\varepsilon_{\max }$ was set to 1 to ensure the maximum probability of exploration when the learning process starts. Figure $2 \mathrm{f}$ shows that with low decr values such as $1 / 1000$ and $1 / 2000$, the DQN agent requires more episodes to converge to a minimized reward than when using a high value such as $1 / 400$. Figure $2 \mathrm{~g}$ shows that a mini-batch $b s=15$ produces a slight improvement in convergence than values $b s=30$ and 45. The parameter tup indicating the number of steps after which the weights and biases of the Target NN are updated. Figure $2 \mathrm{~h}$ shows a slightly improvement when $t u p=100$ than when tup is set to either 300 or 500 .

\section{E. Performance Analysis}

Next, the DRSIR algorithm is compared to that of RSIR and the variations of the Dijkstra's algorithm. The results are averaged every hour; the figures also show the traffic generated per hour. 


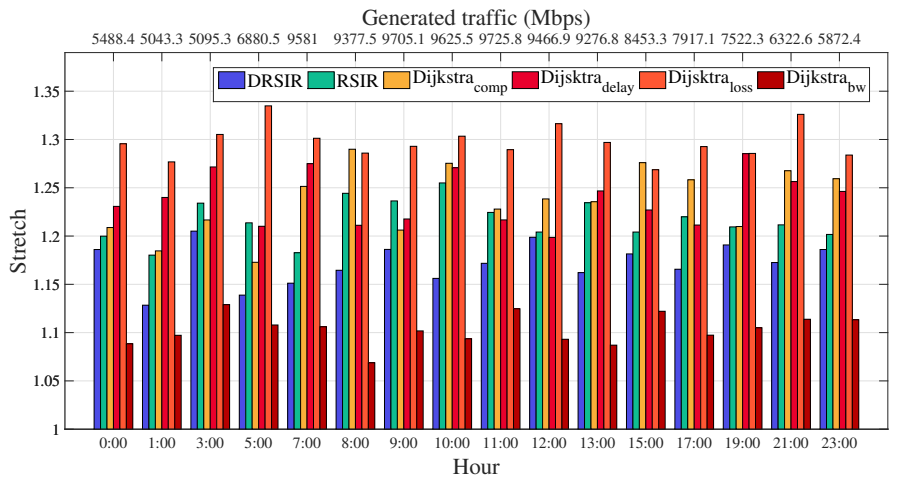

(a) Mean stretch throughout the day

Generated traffic (Mbps)

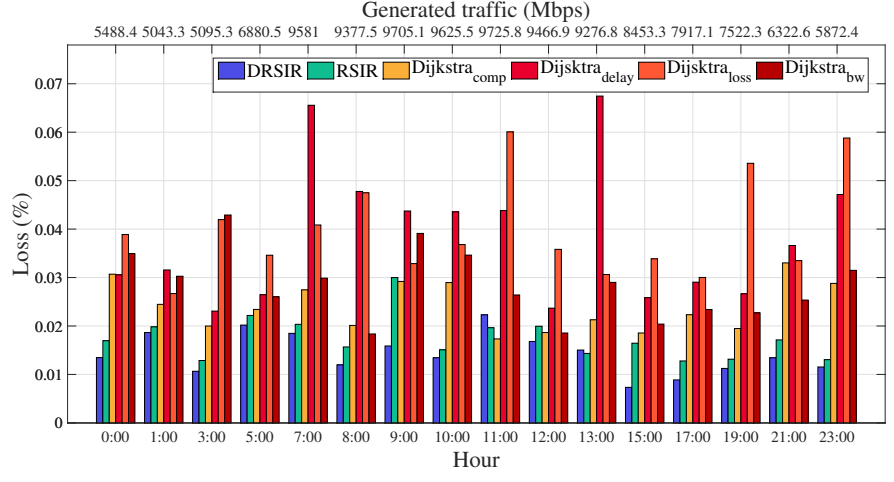

(c) Mean loss throughout the day

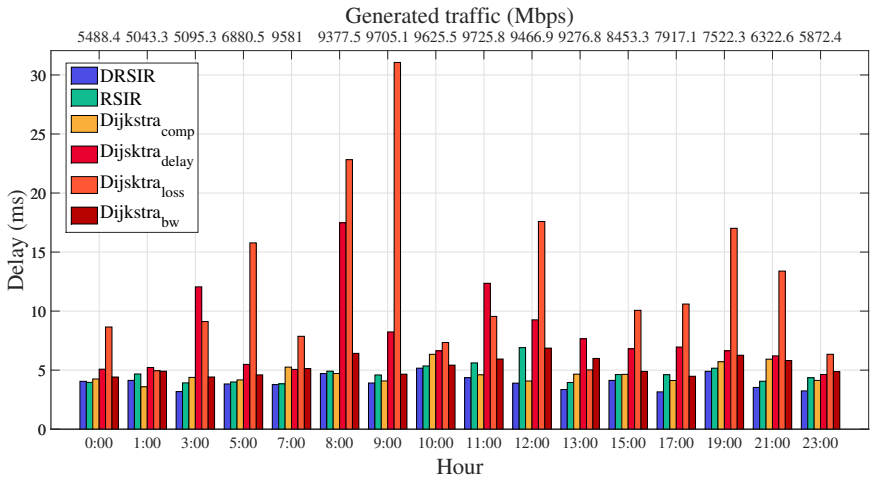

(b) Mean delay throughout the day Generated traffic (Mbps)

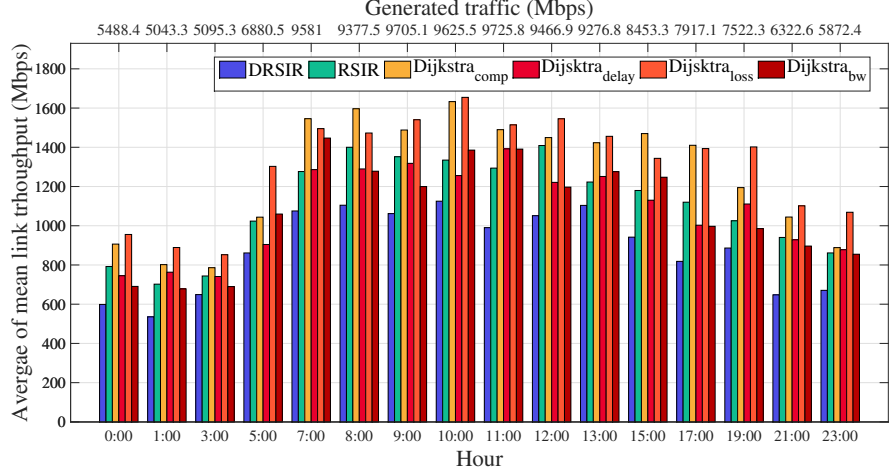

(d) Mean link throughput throughout the day

Fig. 3: Performance metrics resulting from the DRSIR for 23-node topology

1) Results for a 23-node topology: Figure 3 shows the stretch, link delay, loss ratio, and link throughput produced by DRSIR, RSIR, and the variations of the Dijkstra's algorithm for a 23-node topology. Figure 3 a shows the values of the mean stretch averaged over all paths. The paths chosen by the DRSIR present a smaller stretch value than those produced by

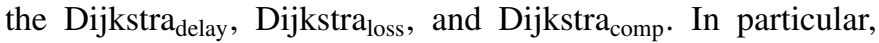
we observe that DRSIR selects a larger number of shorter paths than do the three other algorithms; stretch values $5 \%$, $10 \%$, and $5 \%$ smaller than those obtained by the Dijkstra ${ }_{\text {delay }}$, Dijkstra $_{\text {loss, }}$, and Dijkstra ${ }_{\text {comp }}$ algorithms, respectively. Moreover, the paths produced by the DRSIR are shorter (at most $8 \%$ ) than those produced by the RSIR. The results also show that the DRSIR indicates paths with stretch values slightly higher $(6 \%)$ than those produced by the Dijkstrabw.

Figure $3 \mathrm{~b}$ presents the mean link delay produced by DRSIR, RSIR, and the variations of the Dijkstra's algorithm. The mean link delay produced by the DRSIR algorithm are, on average, $14 \%, 43 \%, 59 \%, 25 \%$, and $15 \%$, at most, $44 \%, 73 \%$, $87 \%, 44 \%$, and $40 \%$ lower than those given by those of the RSIR, Dijkstra ${ }_{\text {delay }}$, Dijkstra ${ }_{\text {loss }}$, Dijkstra ${ }_{b w}$, and Dijkstra ${ }_{\text {comp }}$ algorithms, respectively. The mean link delay results show that the DRSIR algorithm tends to choose less congested paths than do the RSIR and Dijkstra's algorithms; therefore, the delay obtained using the DRSIR is less than that given by the RSIR and the four Dijkstra's variations.

Figure $3 \mathrm{c}$ reveals that the mean loss values produced by the DRSIR algorithm are lower than those given by the RSIR, Dijkstra delay , Dijkstra $a_{\text {loss }}$, Dijkstra ${ }_{b w}$, and Dijkstra $a_{\text {comp. }}$.
In particular, the values produced by the DRSIR algorithm are, on average, $51 \%$ and, at most, $73 \%$ lower than those obtained by the variations of the Dijkstra's algorithm, and on average $17 \%$ and, at most, $55 \%$ lower than that produced by the RSIR algorithm. Results show that Dijkstra's algorithm variations usually select longer routes and more frequently use lowcapacity links, causing traffic concentration and congestion in these links.

Figure 3d shows the mean link throughput throughout the day. DRSIR uses a higher number of paths less utilized than do the RSIR, Dijkstra ${ }_{\text {delay }}$, Dijkstra ${ }_{\text {loss }}$, Dijkstra ${ }_{b w}$, and Dijkstra $_{\text {comp }}$ algorithms. The link throughput is consequently lower than those produced by RSIR and the Dijkstra's variations most of the time with a lower mean delay and loss. The link throughput is on average $20 \%, 18 \%, 33 \%, 18 \%$ and $30 \%$, and at most, $31 \%, 30 \%, 41 \%, 29 \%$ and $42 \%$, lower than those produced by the RSIR, Dijkstra delay, Dijkstra $a_{\text {loss, }}$, bw and Dijkstra $_{\text {comp }}$ algorithms, respectively.

2) Results for a 48-node topology: Figure 4 presents the results for a 48-node topology. Figure 4a shows the mean stretch computed for all the paths found by DRSIR, RSIR, and the variations of the Dijkstra's algorithm. The results evince that DRSIR finds a larger number of shorter paths than do the Dijkstra $_{\text {delay }}$, Dijkstraloss, and Dijkstra comp $_{\text {algorithms for this }}$ topology. The DRSIR algorithm indicates paths with stretch values $23 \%, 7 \%$, and $17 \%$ smaller than those obtained by the Dijkstra $_{\text {delay }}$, Dijkstra $_{\text {loss }}$, and Dijkstra ${ }_{\text {comp }}$ algorithms, respectively. The DRSIR algorithm finds paths with stretch values slightly higher $(<1 \%)$ than those produced by Dijkstra $a_{b w}$. 


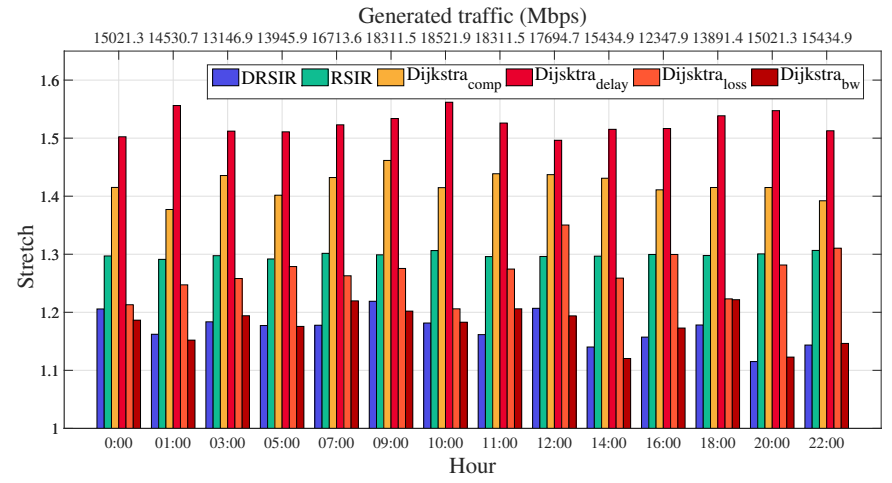

(a) Mean stretch throughout the day

Generated traffic (Mbps)

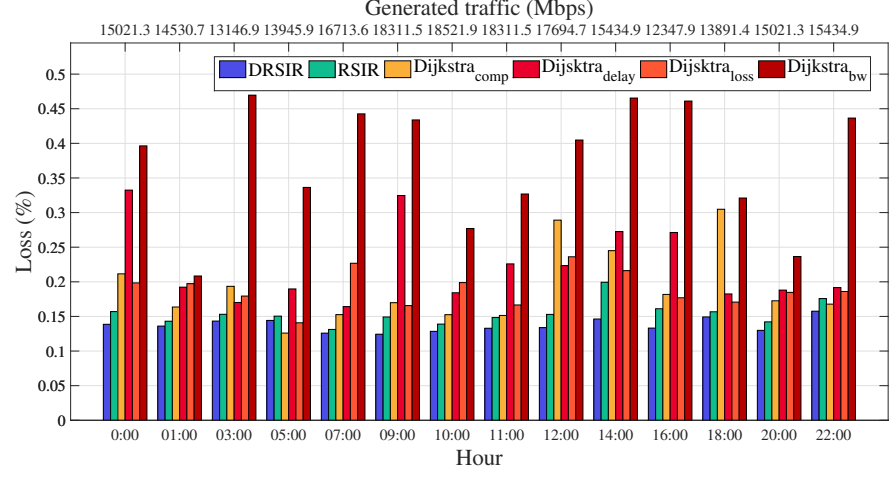

(c) Mean loss throughout the day

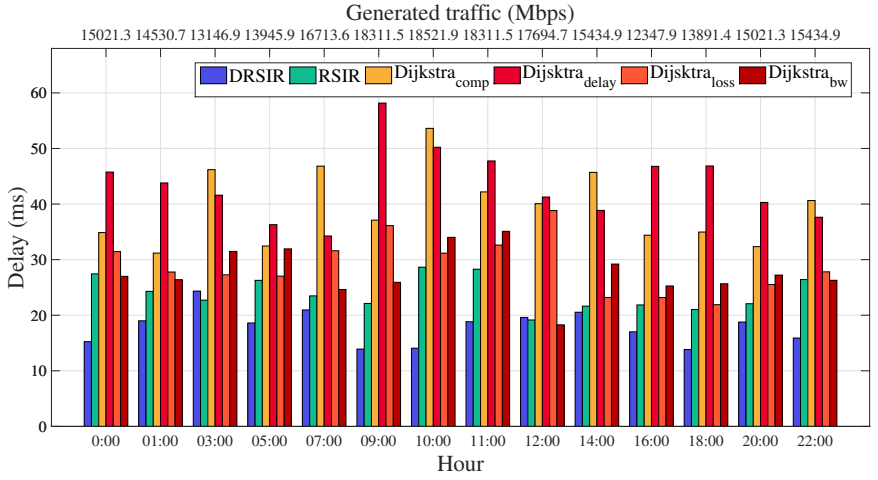

(b) Mean delay throughout the day Generated traffic (Mbps)

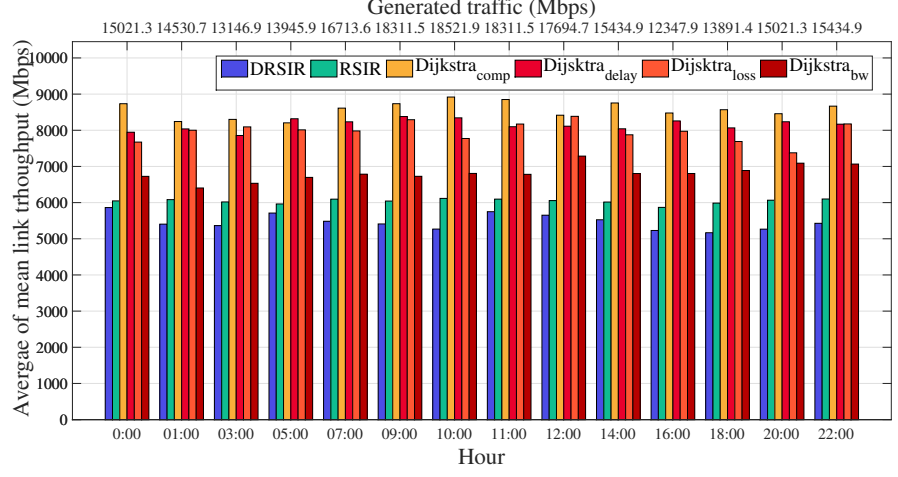

(d) Mean link throughput throughout the day

Fig. 4: Performance metrics resulting from the DRSIR for 48-node topology

Moreover, the paths produced by the DRSIR algorithm are shorter ( $9 \%$ and at most $14 \%$ ) than those given by the RSIR algorithm.

Figures $4 \mathrm{~b}$ and $4 \mathrm{c}$ present the mean delay and mean loss ratio given by the DRSIR, RSIR, and the four variations of the Dijkstra's algorithm throughout the day. The results show that the DRSIR algorithm produces smaller mean delay and smaller mean loss ratio values than those produced by the RSIR algorithm and the four variations of the Dijkstra's algorithm. The mean delay produced by DRSIR are, on average, $24 \%$, $57 \%, 36 \%, 33 \%$, and $54 \%$ lower than the mean delay values produced by the RSIR, Dijkstradelay, Dijkstraloss, Dijkstra ${ }_{b w}$, and Dijkstra ${ }_{\text {comp }}$ algorithms, respectively. The mean loss ratio values observed with the DRSIR algorithm are on average $36 \%$ and at most $57 \%$ lower than those produced by the four variations of the Dijkstra's algorithm, and on average $10 \%$ and at most $26 \%$ lower than those obtained with the RSIR algorithm.

Figure $4 \mathrm{~d}$ presents the values of mean link throughput throughout the day. The results show that the link throughput produced by the DRSIR algorithm is at most $13 \%, 36 \%$, $34 \%, 25 \%$, and $40 \%$ lower than those produced by the RSIR, Dijkstra delay $_{\text {, Dijkstra }}$ loss, Dijkstra bw $_{\text {, }}$, and Dijkstra comp $_{\text {com }}$ algorithms, respectively. The paths chosen by DRSIR include more less utilized links than do the paths chosen by the RSIR and variations of the Dijkstra's algorithm.

3) Topology Change Analysis: When a topology change occurs, the DRSIR algorithm detects the change, calculates new routes, and installs them. For DRSIR, the setup time $t_{\text {chad }}$ is $1 s$. The DQN agent computes all routes for the 23-node and 48-node topologies in $t_{\text {lear }}=5.3 \mathrm{~s}$ and 7, $2 \mathrm{~s}$ (the average execution times of Algorithm 1 for each topology), respectively. The Installation Module spends on average $t_{\text {inst }}=1.6 \mathrm{~s}$ to update the flow entries. Thus, the DRSIR algorithm takes on average $t_{\text {chad }}+t_{\text {lear }}+t_{\text {inst }}=7.9 \mathrm{~s}$ and $9.8 \mathrm{~s}$ to handle a change in the 23-node and 48-node topologies, respectively. In comparison with the Routing Information Protocol (RIP), which would typically take $30 s$ to handle a topological change. The DRSIR algorithm requires a shorter response time due to the employment of a centralized controller with a global view of the network, the generalization provided by the NNs, and the adoption of a network-state routing approach with metrics at the path-level. The OSPF reaction to topological changes when considering a minimum hello-interval $=1 s$ and an spf-delay $=1 s$ is approximately $9 s(5 s+$ the average execution time of the Dijkstra's algorithm), which is slightly lower than the time the DRSIR algorithm takes. The RL-agent of RSIR (using path-state metrics) computes all routes for the 23 and 48-node topologies in $2.8 s$ and $4.7 s$, respectively; thus, the RSIR algorithm takes on average $5.4 \mathrm{~s}$ and $8.1 \mathrm{~s}$ to handle change in the topologies. Therefore, the DRSIR algorithm obtained a $t_{\text {lear }}$ higher than that achieved by the RSIR algorithm because the DQN agent spends more time training two NNs; however, better performance metrics offset this cost.

4) CPU and Memory Analysis: To compare the performance of DRSIR and RSIR, we also evaluate the consumption of CPU and storage memory of its DQN and Q-learning 
TABLE I: Related Work

\begin{tabular}{|c|c|c|c|c|c|}
\hline Paper & Description & Control & Learning approach & Action & Performance metrics \\
\hline [13] & $\begin{array}{l}\text { QAR: QoS-aware adaptive routing with multi-layer hierarchies intended to } \\
\text { minimize signaling delay in large SDNs }\end{array}$ & $\mathrm{C}$ & RL (Q-learning) & Next-hop & $\begin{array}{l}\text { Delivery delay, packet loss, and hop } \\
\text { count }\end{array}$ \\
\hline$[15]$ & $\begin{array}{l}\text { CRE: a logically centralized Cognitive Routing Engine, based on Random } \\
\text { NNs with RL to find optimal overlay paths. }\end{array}$ & $\mathrm{C}$ & RL (Random NN) & Next-hop & Delay \\
\hline$[14]$ & $\begin{array}{l}\text { Link costs calculation for path computation with OSPF for distributed } \\
\text { routing in SDN }\end{array}$ & $\mathrm{D}$ & RL (Q-learning) & Link weight values & Delay and jitter \\
\hline$[16]$ & $\begin{array}{l}\text { An approach called SDCoR for choosing the proper traditional routing } \\
\text { algorithm regarding changes in the Internet of Vehicles (IoV) environment }\end{array}$ & $\mathrm{D}$ & RL (Q-learning) & Routing Protocol & Delivery delay, delivery ratio \\
\hline$[17]$ & $\begin{array}{l}\text { A routing protocol for Wireless Sensor Networks (WSN) based on dis- } \\
\text { tributed RL that learns the path to the sink node }\end{array}$ & $\mathrm{D}$ & RL (Q-learning) & Next-hop & Node lifetime and energy-efficiency \\
\hline$[18]$ & $\begin{array}{l}\text { RSIR: An RL-based approach for intelligent routing in SDN based on } \\
\text { link-state metrics }\end{array}$ & $\mathrm{C}$ & RL (Q-learning) & Next-hop & Link stretch, delay, loss and throughput \\
\hline$[20]$ & $\begin{array}{l}\text { A learning agent based on DRL that optimizes routing to minimize network } \\
\text { delay }\end{array}$ & $\mathrm{C}$ & DRL (DDPG) & Link weight values & Network delay \\
\hline [21] & $\begin{array}{l}\text { A DRL-based mechanism to achieve routing optimization called DROM } \\
\text { in SDN }\end{array}$ & $\mathrm{C}$ & DRL (DDPG) & Link weight values & Delay \\
\hline$[22]$ & $\begin{array}{l}\text { NetworkAI: A network architecture using network monitoring technologies } \\
\text { and artificial intelligence to generate control policies }\end{array}$ & $\mathrm{C}$ & DRL (DQN) & Not reported & Delay \\
\hline [23] & $\begin{array}{l}\text { A DRL-based agent with convolutional neural networks in the context of } \\
\text { KDN designed to enhance the performance of QoS-aware routing }\end{array}$ & $\mathrm{C}$ & DRL (DDPG) & Link weight values & Delay, loss and qualified flows \\
\hline$[24]$ & $\begin{array}{l}\text { Two DQN-based algorithms designed to reduce the network congestion } \\
\text { probability with a short transmission path }\end{array}$ & $\mathrm{C}$ & DRL (DQN) & Next-hop & Throughput \\
\hline$[25]$ & Tide: A time-relevant DRL network control architecture to optimize routing & $\mathrm{C}$ & DRL (DDPG) & Link weight values & Delay \\
\hline [26] & $\begin{array}{l}\text { A DRL-based routing algorithm to manage multiple service requests of } \\
\text { crowd distribution in smart city sectors }\end{array}$ & $\mathrm{C}$ & DRL (DQN) & Next-hop & $\begin{array}{l}\text { Service access delay, successful ac- } \\
\text { cess rate, network usage }\end{array}$ \\
\hline [27] & $\begin{array}{l}\text { DRL-THSA: A Two-hops state-aware routing strategy based on DRL for } \\
\text { LEO satellite networks }\end{array}$ & $\mathrm{D}$ & DRL (DQN) & Next-hop & Delay, throughput, drop rate \\
\hline [28] & $\begin{array}{l}\text { A DRL-based scheme to enable intelligent Service Function Chaining } \\
\text { (SFC) routing decision-making in dynamic network conditions }\end{array}$ & $\mathrm{C}$ & DRL (PPO) & Link weight values & Path delay and link usage \\
\hline$[29]$ & DQSP: A DRL-based QoS-aware secure routing approach for SDN-IoT & $\mathrm{C}$ & DRL (DDPG) & Next-hop & $\begin{array}{l}\text { Packet delivery rate, attacked node } \\
\text { use probability }\end{array}$ \\
\hline$[30]$ & $\begin{array}{l}\text { A deep multi-agent DRL approach for packet routing to avoid network } \\
\text { congestion }\end{array}$ & $\mathrm{D}$ & DRL (DQN) & Next-hop & Path delay and link usage \\
\hline DRSIR & $\begin{array}{l}\text { A DRL-based solution for intelligent routing in SDN based on path-state } \\
\text { metrics }\end{array}$ & $\mathrm{C}$ & DRL (DQN) & Path Selection & Link stretch, delay, loss and throughput \\
\hline
\end{tabular}

C: Centralized and D: Distributed

agents, respectively. Figure 5 shows the results of the evaluation for the 48-node topology, disclosing that the agents do not intensively consume the resources of the Routing Module. Indeed, they only consume $29 \%$ and $32 \%$ of the CPU, and 279 and 287 MBytes of storage memory. Therefore, we can state that the DRSIR and RSIR agents are efficient regarding CPU and storage memory. Moreover, these agents have a constant consumption of RAM (approximately $2.5 \%$ ).

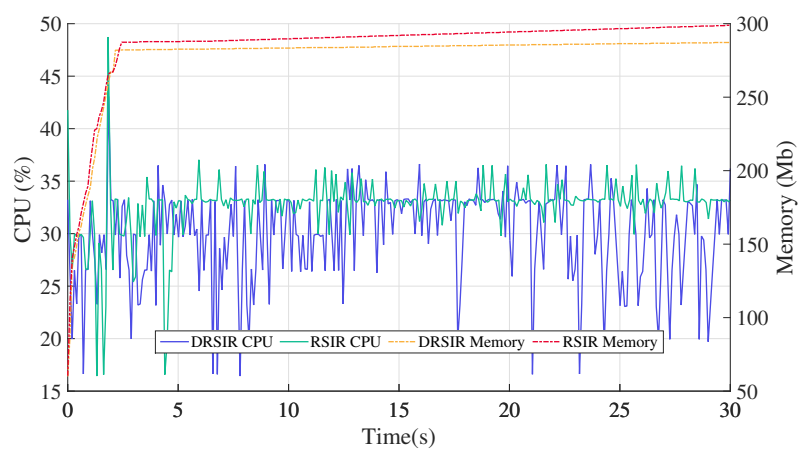

Fig. 5: CPU and Memory used by the agents of DRSIR and RSIR

\section{Comparative Analysis}

Table I briefly summarizes the work related to DRSIR by showing the type of control routing employed, the employed RL/DRL type of learning approach, the RL/DRL adopted action, and the metrics evaluated. The work in [13]-[17] explored RL techniques, such as Q-learning, in SDN either to employ actions for choosing the proper routing protocol within the environment state or at the next-hop node when building a routing path. In the following paragraphs, we compare DRSIR with the routing solutions proposed in [20]-[30], which employed DRL techniques for routing in SDN. Such a comparison is conducted regarding the use of the traditional routing protocols, learning approach and used technique, type of control routing, and type of employed action.

Dependence on Traditional Routing Protocols. Although DRL does not require labeled datasets for agents training, the solutions in [20], [21], [23], [28] depend on traditional routing protocols for computing paths since their agents do not learn to build or select the best path. In these papers, agents that learn to optimize link weight values and use traditional routing protocols (e.g., Dijkstra) to compute paths based on optimized weights are commonly deployed. The work in [22], [24]-[27], [29], [30] and the DRSIR algorithm learn to select the next hop in a path or the end-to-end path for each pair of nodes.

Learning Approach. The DRSIR and the papers [20]- 
[30] employ model-free and off-policy algorithms by learning either a value function or a policy. Specially, the work in [22], [24], [26], [30], and DRSIR employed DQN, which is proper for routing problems where the MDP defines a discrete action space. The works in [20], [21], [23], [25], [27]-[29] employed actor-critic algorithms [34] such as the DDPG (Deep Deterministic Policy Gradient) and PPO (Proximal Policy Optimization) algorithms which are useful for continuous action spaces (e.g., selecting link weights values for each link in a network). Unlike the DQN, the output of the DDPG algorithm is not discretized as a set of fixed actions but rather defined to return a real number/vector. Continuous actions are more challenging learning than discrete actions, and discretizing them can improve performance notably [61].

Routing Control. DRSIR and the works in [20]-[26], [28], [29] leverage the global network view offered by SDN to deploy centralized routing strategies that use either the Knowledge Plane or the Control Plane to compute paths and properly install flows in the tables of the forwarding devices. In contrast, the solutions introduced in [27] and [30] deploy routing strategies in a distributed fashion so that routing nodes turn themselves into learning entities that makes local routing decisions based on information learned from the environment. These routing solutions can generate signaling overhead in the Data Plane that can contribute to network congestion.

Action. The action spaces used in the papers reviewed are commonly link and hop-based. Specifically, we identified two types of actions for the routing solutions: setting up the link weight values of a topology representing the underlying network [20], [21], [23], [25], [28], and selecting the nexthop node when building a routing path [24], [26], [27], [29], [30]. Both kinds of action can limit the solutions for the use of external routing algorithms such as the Dijkstra's algorithm. Furthermore, the performance of an end-to-end path performance can only be satisfied when all hops are appropriately chosen; the performance can drop significantly when any hop is wrongly selected by a link/hop-based routing scheme. Unlike what is found in other papers, the DRSIR algorithm optimizes routing decisions by employing path-state metrics as features in the learning process, meaning that the DRSIR agent directly uses knowledge of the paths instead of extracting information from the link-state to choose the nexthop node or update link weight values.

\section{CONCLUSIONS}

In this paper, we have introduced DRSIR, an approach which employs DRL for routing in SDN. The DRSIR uses Deep Q-learning, a model-free algorithm that makes the DRLagent find the appropriate routing policy without making any assumption about the dynamics of the environment. DRSIR learns while transitioning over states and actions without prior knowledge about the underlying network. The DRL-based routing algorithm of the DRSIR produces routes for every pair of source-destination nodes in a network. It prioritizes paths with high available bandwidth and low delay and loss as the best routing option based on path-state metrics. The results showed that most of the time the DRSIR indicates shorter and less congested paths than those indicated by the RSIR and the four versions of Dijkstra's algorithm. As a result, the mean delay and loss produced by DRSIR are lower than that the given by the RSIR and Dijkstra's variations. It is noteworthy that the DRSIR does not require previously labeled data representing the system under consideration for training the DRL-based agent. The acquisition of labeled training datasets has high computational complexity and makes the routing solutions dependent on traditional routing protocols to build such datasets. The results presented in this paper encourage the use of the DRSIR as a feasible and practical strategy to carry out routing in SDN.

For future work, we intend to explore multi-level RL schemes to deal with centralized control challenges such as scalability on larger-scale networks. We also intend to improve the set up of the learning parameters in order to make the agent self-configurable.

\section{ACKNOWLEDGMENT}

The authors would like to thank CAPES, and the Sao Paulo Research Foundation (FAPESP) for grant \#19/03268-0 and 2015/24494-8.

\section{REFERENCES}

[1] A. Rego, S. Sendra, J. M. Jimenez, and J. Lloret, "Ospf routing protocol performance in software defined networks," in IEEE SDS, 2017, pp. $131-136$.

[2] D. Gopi, S. Cheng, and R. Huck, "Comparative analysis of sdn and conventional networks using routing protocols," in IEEE CITS, 2017, pp. 108-112.

[3] J. Park, J. Hwang, and K. Yeom, "Nsaf: An approach for ensuring application-aware routing based on network qos of applications in sdn," Mobile Information Systems, 2019.

[4] A. Shirmarz and A. Ghaffari, "An adaptive greedy flow routing algorithm for performance improvement in software-defined network," International Journal of Numerical Modelling: Electronic Networks, Devices and Fields, vol. 33, no. 1, 2020.

[5] Y.-C. Wang and S.-Y. You, "An efficient route management framework for load balance and overhead reduction in sdn-based data center networks," IEEE TNSM, vol. 15, no. 4, pp. 1422-1434, 2018.

[6] C.-C. Chuang, Y.-J. Yu, and A.-C. Pang, "Flow-aware routing and forwarding for sdn scalability in wireless data centers," IEEE TNSM, vol. 15, no. 4, pp. 1676-1691, 2018.

[7] N. Kato, Z. M. Fadlullah, B. Mao, F. Tang, O. Akashi, T. Inoue, and K. Mizutani, "The deep learning vision for heterogeneous network traffic control: Proposal, challenges, and future perspective," IEEE Wireless Communications, vol. 24, no. 3, pp. 146-153, 2016.

[8] Z. M. Fadlullah, F. Tang, B. Mao, N. Kato, O. Akashi, T. Inoue, and K. Mizutani, "State-of-the-art deep learning: Evolving machine intelligence toward tomorrow's intelligent network traffic control systems," IEEE Communications Surveys \& Tutorials, vol. 19, no. 4, pp. 2432 2455, 2017.

[9] S. Chaudhary and R. Johari, "Oruml: Optimized routing in wireless networks using machine learning," IJCS, 2020.

[10] A. Serhani, N. Naja, and A. Jamali, "Aq-routing: mobility-, stabilityaware adaptive routing protocol for data routing in manet-iot systems," Cluster Computing, vol. 23, no. 1, pp. 13-27, 2020.

[11] Y. Liu, J. Zhang, W. Li, Q. Wu, and P. Li, "Load balancing oriented predictive routing algorithm for data center networks," Future Internet, vol. 13, no. 2, p. 54, 2021.

[12] F. Alhaidari, S. H. Almotiri, M. A. K. Mohammed Al Ghamdi, A. Rehman, S. Abbas, K. M. Khan, and A. ur Rahman, "Intelligent software-defined network for cognitive routing optimization using deep extreme learning machine approach," Computers, Materials \& Continua, vol. 67, no. 1, pp. 1269-1285, 2021.

[13] S.-C. Lin, I. F. Akyildiz, P. Wang, and M. Luo, "Qos-aware adaptive routing in multi-layer hierarchical software defined networks: a reinforcement learning approach," in IEEE SCC, 2016, pp. 25-33. 
[14] S. Sendra, A. Rego, J. Lloret, J. M. Jimenez, and O. Romero, "Including artificial intelligence in a routing protocol using software defined networks," in IEEE ICC Workshops, 2017, pp. 670-674.

[15] P. Liu, "The random neural network and its learning process in cognitive packet networks," in IEEE ICNC, 2013, pp. 95-100.

[16] C. Wang, L. Zhang, Z. Li, and C. Jiang, "Sdcor: Software defined cognitive routing for internet of vehicles," IEEE Internet of Things Journal, vol. 5, no. 5, pp. 3513-3520, 2018

[17] S. Bouzid, Y. Serrestou, K. Raoof, and M. Omri, "Efficient routing protocol for wireless sensor network based on reinforcement learning," in IEEE ATSIP, 2020, pp. 1-5.

[18] D. M. Casas-Velasco, O. M. C. Rendon, and N. L. S. da Fonseca, "Intelligent routing based on reinforcement learning for software-defined networking," IEEE TNSM, vol. 18, no. 1, pp. 870-881, 2021.

[19] K. Arulkumaran, M. P. Deisenroth, M. Brundage, and A. A. Barath, "A brief survey of deep reinforcement learning," IEEE Signal Processing Magazine, vol. 34, pp. 26-38, 2017.

[20] G. Stampa, M. Arias, D. Sanchez-Charles, V. Muntés-Mulero, and A. Cabellos, "A deep-reinforcement learning approach for softwaredefined networking routing optimization," CoRR, 2017.

[21] C. Yu, J. Lan, Z. Guo, and Y. Hu, "Drom: Optimizing the routing in software-defined networks with deep reinforcement learning," IEEE Access, vol. 6, pp. 64 533-64 539, 2018.

[22] H. Yao, T. Mai, X. Xu, P. Zhang, M. Li, and Y. Liu, "Networkai: An intelligent network architecture for self-learning control strategies in software defined networks," IEEE Internet of Things Journal, vol. 5, no. 6, pp. 4319-4327, 2018.

[23] T. A. Q. Pham, Y. Hadjadj-Aoul, and A. Outtagarts, "Deep reinforcement learning based qos-aware routing in knowledge-defined networking," in Springer Qshine, 2018, pp. 14-26.

[24] R. Ding, Y. Xu, F. Gao, X. Shen, and W. Wu, "Deep reinforcement learning for router selection in network with heavy traffic," IEEE Access, vol. 7, pp. 37 109-37 120, 2019.

[25] P. Sun, Y. Hu, J. Lan, L. Tian, and M. Chen, "Tide: Time-relevant deep reinforcement learning for routing optimization," Future Generation Computer Systems, vol. 99, pp. 401-409, 2019.

[26] L. Zhao, J. Wang, J. Liu, and N. Kato, "Routing for crowd management in smart cities: A deep reinforcement learning perspective," IEEE Communications Magazine, vol. 57, no. 4, pp. 88-93, 2019.

[27] C. Wang, H. Wang, and W. Wang, "A two-hops state-aware routing strategy based on deep reinforcement learning for leo satellite networks," Electronics, vol. 8, no. 9, p. 920, 2019.

[28] Z. Ning, N. Wang, and R. Tafazolli, "Deep reinforcement learning for nfv-based service function chaining in multi-service networks : Invited paper," in IEEE HPSR, 2020, pp. 1-6.

[29] X. Guo, H. Lin, Z. Li, and M. Peng, "Deep-reinforcement-learning-based qos-aware secure routing for sdn-iot," IEEE Internet of Things Journal, vol. 7, no. 7, pp. 6242-6251, 2019.

[30] R. Ding, Y. Yang, J. Liu, H. Li, and F. Gao, "Packet routing against network congestion: A deep multi-agent reinforcement learning approach," in IEEE ICNC, 2020, pp. 932-937.

[31] N. C. Luong, D. T. Hoang, S. Gong, D. Niyato, P. Wang, Y.-C Liang, and D. I. Kim, "Applications of deep reinforcement learning in communications and networking: A survey," IEEE Communications Surveys \& Tutorials, vol. 21, no. 4, pp. 3133-3174, 2019.

[32] Z. Mammeri, "Reinforcement learning based routing in networks: Review and classification of approaches," IEEE Access, vol. 7, pp. 5591655950, 2019.

[33] V. François-Lavet, P. Henderson, R. Islam, M. G. Bellemare, an J. Pineau, "An introduction to deep reinforcement learning," Foundations and Trends in Machine Learning, vol. 11, no. 3-4, pp. 219-354, 2018.

[34] R. S. Sutton and A. G. Barto, Reinforcement learning: An introductionsecond edition. Cambridge, Massachusetts, 2018, vol. 1.

[35] I. J. Okonkwo and I. D. Emmanuel, "Comparative study of eigrp and ospf protocols based on network convergence," IJACSA, vol. 11, no. 6 , 2020.

[36] V. Mnih, K. Kavukcuoglu, D. Silver, A. A. Rusu, J. Veness, M. G Bellemare, A. Graves, M. Riedmiller, A. K. Fidjeland, G. Ostrovski et al., "Human-level control through deep reinforcement learning," nature, vol. 518, no. 7540, pp. 529-533, 2015.

[37] L. Liao and V. C. Leung, "Lldp based link latency monitoring in software defined networks," in IEEE CNSM, 2016, pp. 330-335.

[38] Z. Shu, J. Wan, J. Lin, S. Wang, D. Li, S. Rho, and C. Yang, "Traffic engineering in software-defined networking: Measurement and management," IEEE access, vol. 4, pp. 3246-3256, 2016. [39] Ryu Project
cessed: Meam, "Ryu application API," [Achttps://ryu.readthedocs.io/en/latest/ryu_app_api.html

[40] L. Al Shalabi and Z. Shaaban, "Normalization as a preprocessing engine for data mining and the approach of preference matrix," in IEEE DepCoS, 2006, pp. 207-214.

[41] Z. Yang, Y. Xie, and Z. Wang, "A theoretical analysis of deep qlearning," in PMLR Learning for Dynamics and Control, 2020, pp. 486489

[42] S. R. White, J. E. Hanson, I. Whalley, D. M. Chess, and J. O. Kephart, "An architectural approach to autonomic computing," in IEEE ICAC, 2004, pp. 2-9.

[43] D. Bienstock, G. Muñoz, and S. Pokutta, "Principled deep neural network training through linear programming," in ICLR, 2019.

[44] P. T. Kirstein, "European international academic networking: A 20 year perspective." in TERENA Networking Conference, 2004.

[45] S. Koenig and R. G. Simmons, "Complexity analysis of real-time reinforcement learning," in AAAI, 1993, pp. 99-107.

[46] R. L. S. De Oliveira, C. M. Schweitzer, A. A. Shinoda, and L. R. Prete, "Using mininet for emulation and prototyping software-defined networks," in IEEE COLCOM, 2014, pp. 1-6.

[47] A. L. Stancu, S. Halunga, A. Vulpe, G. Suciu, O. Fratu, and E. C. Popovici, "A comparison between several software defined networking controllers," in IEEE TELSIKS, 2015, pp. 223-226.

[48] ONF, "Openflow switch specification v1.5.0," Open Network Foundation, Technical Specification TS-020, December 2014. [Online]. Available: https://www.opennetworking.org/images/stories/downloads/sdnresources/onf-specifications/openflow/openflow-switch-v1.5.0.noipr.pdf

[49] M. Abadi, P. Barham, J. Chen, Z. Chen, A. Davis, J. Dean, M. Devin, S. Ghemawat, G. Irving, M. Isard, M. Kudlur, J. Levenberg, R. Monga, S. Moore, D. G. Murray, B. Steiner, P. Tucker, V. Vasudevan, P. Warden, M. Wicke, Y. Yu, and X. Zheng, "Tensorflow: A system for large-scale machine learning," in USENIX OSDI, 2016, pp. 265-283.

[50] T. E. Oliphant, A guide to NumPy. Trelgol Publishing USA, 2006 , vol. 1.

[51] W. McKinney, "Data structures for statistical computing in python," in SciPy, S. van der Walt and J. Millman, Eds., 2010, pp. 51-56.

[52] D. M. Casas-Velasco, O. M. Caicedo, and N. L. S $\mathrm{Da}$ Fonseca, "Routing based on (deep) reinforcement learning for software-defined networking," in https://github.com/danielaCasasv/DRSIR_DRL_routing_approach_for SDN, 2021.

[53] C. Werle, S. Mies, and M. Zitterbart, "On benchmarking routing protocols," in IEEE ICON, 2011, pp. 305-310.

[54] S. Uhlig, B. Quoitin, J. Lepropre, and S. Balon, "Providing public intradomain traffic matrices to the research community," SIGCOMM Computer Communication Review, vol. 36, no. 1, pp. 83-86, 2006.

[55] P. Tune and M. Roughan, "Spatiotemporal traffic matrix synthesis," in SIGCOMM. ACM, 2015, p. 579-592.

[56] D. P. Kingma and J. Ba, "Adam: A method for stochastic optimization," in ICLR. Ithaca, 2015 .

[57] E. Okewu, P. Adewole, and O. Sennaike, "Experimental comparison of stochastic optimizers in deep learning," in Springer ICCSA, 2019, pp. 704-715.

[58] X. Glorot and Y. Bengio, "Understanding the difficulty of training deep feedforward neural networks," in PMLR AISTATS, vol. 9, 2010, pp. 249256.

[59] I. Goodfellow, Y. Bengio, A. Courville, and Y. Bengio, Deep learning. MIT press Cambridge, 2016, vol. 1, no. 2.

[60] K. Hornik, M. Stinchcombe, H. White et al., "Multilayer feedforward networks are universal approximators." Neural networks, vol. 2, no. 5, pp. 359-366, 1989

[61] A. Kanervisto, C. Scheller, and V. Hautamäki, "Action space shaping in deep reinforcement learning," in IEEE CoG, 2020, pp. 479-486.

Daniela M. Casas-Velasco (GS'13) is pursuing her Ph.D degree in Computer Science at the State University of Campinas, Campinas, Brazil.

Oscar M. Caicedo (GS'11-M'15-SM'20) is a Full Professor at the Departamento de Telematica, Universidad del Cauca.

Nelson L. S. da Fonseca (M'88-SM'01) is a Full Professor with the Institute of Computing, State University of Campinas, Brazil. 